\title{
Mitochondrial dynamics in neurodegenerative diseases
}

\author{
Adriana Alarcón-Aguilar, ${ }^{1}$ Paola Maycotte-González,, Paulina Cortés-Hernández, ${ }^{2}$ \\ Norma Edith López-Diazguerrero ${ }^{1}$ and Mina Königsberg ${ }^{1 *}$ \\ ${ }^{1}$ Universidad Autónoma Metropolitana, Department of Health Sciences, Ciudad de México, Mexico; ${ }^{2}$ Instituto Mexicano del Seguro Social, Centro \\ de Investigación Biomédica de Oriente, Puebla, Mexico
}

\begin{abstract}
Neurodegenerative diseases are a group of heterogeneous diseases characterized by a gradual, progressive and selective decrease in nervous system functions. The etiology of these pathologies remains unknown; however, mitochondrial function has been proposed as a common factor that could be involved in the establishment of these diseases, owing to the high energy requirement neurons have in order to carry out their physiological functions. Mitochondria are extremely dynamic organelles that can change their morphology and function in response to different physiological stimuli and, for this reason, mitochondrial dynamics have started being studied as one of cell survival main regulators. This event comprises different processes, such as the generation of new mitochondria and their elimination when they are no longer functional, as well as mitochondrial fusion and fission processes and the traffic of these organelles within the cellular environment. All these processes are highly regulated, and their main purpose is optimal functionality of mitochondria and cellular homeostasis.
\end{abstract}

KEY WORDS: Mitochondria. Neurodegenerative diseases. Oxidative stress. Neurons.

\section{Introduction}

Neurodegenerative diseases (NDs) constitute a heterogeneous group characterized by a progressive decrease of physiological and anatomical functions of the nervous system. The most common aging-associated NDs are Alzheimer's (AD), Parkinson's (PD) and Huntington diseases (HD). The etiology of these pathologies still is unknown, but the mitochondrial function has been proposed as a common factor among them because of the high energy requirement neurons have to perform their physiological functions. ${ }^{1,2}$ In addition, the mitochondria also participates in intracellular signaling, reactive oxygen species (ROS) production and apoptosis. ${ }^{3,4}$

The mitochondrion is a highly dynamic organelle that changes its morphology and function in response to different physiological stimuli, and thus mitochondrial dynamics regulates cell survival. ${ }^{5,6}$ Mitochondrial dynamics comprises different processes such as the generation of new mitochondria (mitochondrial biogenesis), elimination thereof (mitophagy) and, particularly, fusion, fission, transportation and distribution within the cell (traffic).

\section{Mitochondrial fission and fusion}

The most studied mitochondrial dynamics events are mitochondrial fusion and fission (division). These processes occur continuously and allow functional portions of the mitochondrial network to separate and relocate within the cell for energy production. ${ }^{7,8}$ They are essential for mitochondrial DNA maintenance and distribution, organelle quality control and apoptosis regulation..$^{8,9}$

The central proteins that execute the fusion and fission are highly-preserved GTPases related to the family of dynamins known as dynamin-related proteins (DRPs). ${ }^{7}$
Date of reception: 15-04-2018

Date of acceptance: 28-08-2018

DOI: 10.24875/GMM.M19000270
Gac Med Mex. 2019;155:254-260

Contents available at PubMed www.gacetamedicademexico.com 


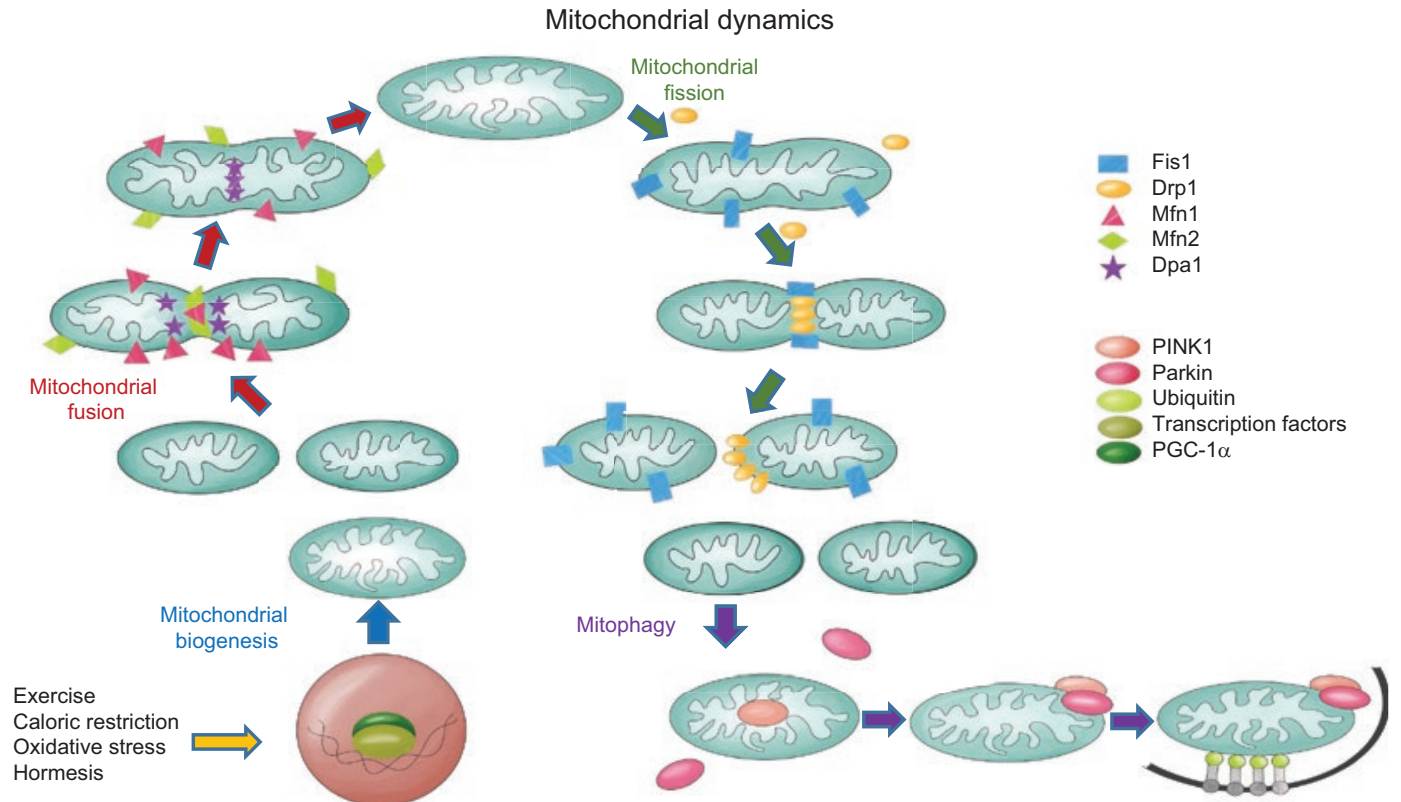

Figure 1. Representation of mitochondrial dynamics and participating proteins. The fusion pathway is exemplified by the red color, fission with green, while biogenesis is represented with blue and mitophagy with purple.

Dynamin-related protein 1 (Drp1) drives mitochondrial fission. Drp1 is a cytosolic protein that is recruited and anchored in the mitochondrial outer membrane (MOM) thanks to the presence of several transmembrane proteins such as fission protein 1 (Fis 1). ${ }^{10}$ Drp1 is assembled to form a spiral composed of hundreds of Drp1 monomers that roll up and wrap thin portions of the mitochondrial tubules, strangling them and simultaneously excising the outer and inner membranes. ${ }^{10}$

During the fusion, GTPases of the DRP family also participate, unlike most intracellular fusion events, which use proteins of the SNARE type. ${ }^{11}$ Mitochondrial fusion occurs in two steps: first, the MOMs of two mitochondria merge through the interaction of mitofusins (Mfn1/Mfn2), which are anchored in these membranes; in a second stage, the optic atrophy-related protein 1 (Opa1) fuses the mitochondrial inner membranes (MIM). ${ }^{12,13}$ These events can be separated due to their different energy requirements: the MIM fusion demands higher GTP concentrations than the MOM fusion, but both occur only among mitochondria that maintain a membrane potential (Figure 1, red and green pathways). The DRPs that participate in these processes are regulated by post-translational modifications..$^{14-21}$

\section{Mitochondrial biogenesis}

Mitochondrial mass can increase in response to various stimuli such as exercise, hormones, cell differentiation and division, oxidative stress, etc. ${ }^{22}$ Due to its endosymbiotic origin, the generation of mitochondria requires concerted expression of two genomes: the nuclear one, which contributes with more than 1000 proteins, and the mitochondrial genome, which in human beings only codes for 13 proteins, all hydrophobic subunits of the respiratory chain (plus 22 tRNA and two rRNA). ${ }^{22,23}$ Given that many mitochondrial proteins are expressed from the nuclear genome, the biogenesis process largely depends on protein import and assembly in mitochondrial compartments $^{24}$ (Figure 1, blue pathway).

The expression of the two genomes is coordinated by PGC-1 $\alpha$ transcriptional coactivator (peroxisome proliferator-activated receptor $1 \alpha$ coactivator), considered the main regulator of mitochondrial biogenesis. ${ }^{25,26}$ PGC-1 $\alpha$ lacks DNA-binding domains, and thus it acts by activating multiple transcription factors, among which nuclear respiratory factors, peroxisome proliferator-activated receptors and estrogen-related receptors stand out, which control oxidative phosphorylation genes, fatty acid oxidation, antioxidant defense, etc. ${ }^{23}$

\section{Mitophagy}

Mitochondria are eliminated through a regulated process called mitophagy, a form of selective macroautophagy. Macroautophagy is the degradation mechanism where a double-membrane vesicle, the autophagosome, is formed around the damaged cell components and then is fused with a lysosome for content degradation. 
Table 1. Characteristics of the three main neurodegenerative diseases

\begin{tabular}{|c|c|c|c|}
\hline Characteristic & Alzheimer & Parkinson & Huntington \\
\hline Origin & Hereditary and environmental & Hereditary and environmental & Hereditary \\
\hline $\begin{array}{l}\text { Disease } \\
\text { manifestations }\end{array}$ & $\begin{array}{l}\text { Memory, learning, consciousness } \\
\text { and language loss }\end{array}$ & $\begin{array}{l}\text { Motor disorders such as bradykinesia } \\
\text { and tremor at rest and stiffness }\end{array}$ & $\begin{array}{l}\text { Motor dysfunction, involuntary } \\
\text { movements, dystonia, cognitive } \\
\text { impairment, intellectual impairment } \\
\text { and emotional disorders }\end{array}$ \\
\hline $\begin{array}{l}\text { Affected regions } \\
\text { of the brain }\end{array}$ & Hippocampus & $\begin{array}{l}\text { Substantia nigra dopaminergic neurons } \\
\text { degeneration }\end{array}$ & $\begin{array}{l}\text { Striate body caudate and putamen, } \\
\text { cerebral cortex, hippocampus, } \\
\text { hypothalamus and subthalamus }\end{array}$ \\
\hline Markers & $\begin{array}{l}\text { Formation of neurofibrillary tangles } \\
\text { and aggregates of } \\
\beta \text {-amyloid peptide fibers }\end{array}$ & $\begin{array}{l}\text { Presence of intracytoplasmic inclusions } \\
\text { (Lewy bodies). Formation of } \beta \text {-amyloid } \\
\text { plaques, } \\
\text { neurofibrillary tangles }\end{array}$ & Presence of mutated huntingtin ( $\mathrm{mHtt}$ ) \\
\hline $\begin{array}{l}\text { Physiological } \\
\text { alterations }\end{array}$ & $\begin{array}{l}\text { - Mitochondrial dysfunction } \\
\text { - Oxidative stress } \\
\text { - Apoptosis } \\
\text { - Synaptic transmission }\end{array}$ & $\begin{array}{l}\text { - Mitochondrial dysfunction } \\
\text { - Oxidative stress } \\
\text { - Apoptosis } \\
\text { - Autophagy via lysosome }\end{array}$ & $\begin{array}{l}\text { - Mitochondrial dysfunction } \\
\text { - Oxidative stress } \\
\text { - Apoptosis }\end{array}$ \\
\hline $\begin{array}{l}\text { Mitochondrial } \\
\text { dynamics }\end{array}$ & $\begin{array}{l}\text { - Mitochondrial fission } \\
\text { - Drp1 } \\
\text { - Mitochondrial fusion } \\
\text { - Mfn2 } \\
\text { - Opa1 }\end{array}$ & $\begin{array}{l}\text { - Mitochondrial fission } \\
\text { - Drp1 } \\
\text { - Mitochondrial fusion } \\
\text { - Mfn1 and Mfn2 } \\
\text { - Opa1 }\end{array}$ & $\begin{array}{l}\text { - Mitochondrial fission } \\
\text { - Drp1 } \\
\text { - Fis1 } \\
\text { - Mitochondrial fusion } \\
\text { - Opa1 } \\
\text { - Mfn1 and Mfn2 } \\
\text { - Tom40 }\end{array}$ \\
\hline $\begin{array}{l}\text { Mitochondrial } \\
\text { biogenesis }\end{array}$ & $-P G C-1 \alpha$ & $-\mathrm{PGC}-1 \alpha$ & $\begin{array}{l}\text { - PGC- } 1 \alpha \\
\text { - TFAM }\end{array}$ \\
\hline Mitophagy & - By Tau intracellular accumulation & $\begin{array}{l}\text { - In general, and with mutations in } \\
\text { PINK1 and Parkin in hereditary PD }\end{array}$ & $\begin{array}{l}\text { - Due to lack of interaction of } \mathrm{Htt} \text { with } \\
\text { p62 to recognize proteins that are } \\
\text { to be ubiquitinated }\end{array}$ \\
\hline
\end{tabular}

When damaged portions of the mitochondrial network cannot generate membrane potential, they fission and are eliminated by mitophagy. ${ }^{27,28}$ The most widely studied mitophagy mechanism is mediated by PINK1 and Parkin proteins. PINK1 kinase (PTEN-induced serine/ threonine kinase) is constantly degraded in healthy mitochondria by protease PARL (presenilins-associated rhomboid-like protein), which is inactivated in depolarized mitochondria, thus stabilizing PINK1 in the MOM. PINK recruits and phosphorylates cytosolic ubiquitin-ligase E3, Parkin, and, in turn, Parkin promotes ubiquitination of several MOM proteins that serve as a signal to recruit the autophagy machinery, thus eliminating damaged mitochondria ${ }^{8,28-30}$ (Figure 1, purple pathway).

\section{Mitochondrial traffic}

Mitochondrial traffic refers to mitochondrial transport and distribution in cells. Particularly, in neurons, mitochondrial concentration is higher in presynaptic regions; mitochondria travel long distances within the neuron to satisfy their high energy requirements. This is accomplished thanks to the coupling between motor and adapter proteins. The best characterized adapter proteins, which bind mitochondria to kinesin heavy chain, are Miro and Milton. Miro is a Rho-GTPase, and in mammals it has two isoforms: Miro-1 and Miro-2. Similarly, there are two Milton orthologues, TRAK1 and TRAK2. ${ }^{31}$

These processes must be regulated in order for cells to be in homeostasis. Alterations in mitochondrial dynamics have been associated with the etiology of several neurodegenerative diseases (Table 1).

\section{Alzheimer's disease}

Alzheimer's disease (AD) is one of the most recurrent neuropathologies in older adults. It has been reported that 60 to $80 \%$ of dementia cases are associated with it, $^{32}$ that it is characterized by progressive loss of neurons in brain regions related to functions such as memory, learning, consciousness and language. Characteristic neuropathological markers include neurofibrillary tangles, intracellular aggregates composed 
of the hyperphosphorylated form of the microtubule-associated Tau protein and senile plates, which are extracellular lesions formed by aggregates of amyloid- $\beta$ peptide fibres. ${ }^{32.33}$

Recently, morphological changes in the brains of patients with $A D$ have been reported to exist, characterized by the presence of fragmented mitochondria, which is associated with Drp1 high activity. Excessive mitochondrial fission contributes to mitochondrial dysfunction and synaptic transmission deficiency, which leads to an increase in neural cell death. ${ }^{34.35}$ In a dual-transgenic AD model (amyloid precursor protein 1/presenilin 1), Drp1 inhibition was observed to prevent mitochondrial fragmentation, loss of mitochondrial membrane potential, ROS production, ATP reduction and synaptic function in neurons treated with $\beta$-amyloid peptide. In addition, Drp1 inhibition significantly improved learning and memory, decreased lipid peroxidation and $\beta$-amyloid deposition. Therefore, mitochondrial fission inhibitors (Drp1 inhibitors) were proposed as a new therapeutic strategy for AD. ${ }^{36}$ This was confirmed in another work carried out in the hippocampus of 12-month mice that overexpressed the Tau protein; an increase in the number of mitochondria with shorter length than normal, increased levels of the Drp1 and Fis1 fission proteins and decreased levels of the Mfn1, Mfn2 and Opa1 fusion proteins were observed. ${ }^{37}$

Mitochondrial biogenesis is also altered in the brains of people with AD. Different studies have shown a reduction in the number of mitochondria in hippocampal neurons in the brains of patients with $A D$ and in M17 cells that overexpress the mutant of the amyloid precursor protein (APPswe). In addition, the levels of PGC-1 $\alpha$, NRF1, NRF2 and TFAM expression are reduced in the hippocampus of people with $A D$ and in APPswe M17 cells, which suggests reduced mitochondrial biogenesis. In fact, the APPswe M17 cells showed a decrease in the mitochondrial DNA/nuclear DNA ratio, correlated with a reduced ATP content. However, taking into account PGC- $1 \alpha$ pleiotropic functions, it is not yet clear whether such changes have a direct influence on mitochondrial dysfunction during AD. ${ }^{38}$

As for mitophagy, the brains of patients with $A D$ and transgenic mice that overexpress the human Tau protein (hTau), were found to show mitophagy deficiencies. hTau overexpression alters the mitochondrial membrane potential, while PINK1 and Parkin levels are decreased. An impairment dependent on the amount of Tau present in the MOM mitochondrial fraction along with its cytoplasmic accumulation were also detected.
These data suggest that hTau intracellular accumulation induces mitophagy deficiency due to its direct insertion in the mitochondrial membrane, which alters the membrane potential, thus affecting the PINK1/Parkin ratio. ${ }^{39}$ Another study with 5xFAD mice that accumulate the $\beta$-amyloid protein reported an increase in Parkin and in LC3II protein recruitment in synaptosomal mitochondria depending on age, as well as increased Drp1 and decreased Mfn2 and Opa1 levels, which points to an increase in mitophagy in synaptosomal mitochondria, which would partially explain the deficiency of synapses in $A D^{40}$

The Miro proteins, involved in mitochondrial transport regulation, have been associated with neurodegenerative and neurodevelopmental disorders, such as in AD. ${ }^{41}$ Although no direct association has been demonstrated between Miro and $A D$, the Miro levels are known to be negatively regulated in the presenilin 1 E280A mutation, which is associated with AD. ${ }^{42} \mathrm{Re}$ cent studies revealed that the brains of patients with $A D$ accumulate defective mitochondria in neurons distal regions, which is correlated with incorrect mitochondrial transport, hindering that damaged mitochondria can be transported to the soma for degradation, which generates inadequate mitophageal clearance; ${ }^{31}$ however, the relationship of mitochondrial traffic defects and AD still needs to be further investigated in order to establish possible treatments.

\section{Parkinson's disease}

PD is the second most common ND and is associated with motor disorders such as bradykinesia, tremor at rest and stiffness. It is characterized by a progressive degeneration of dopaminergic neurons (DAN) in the substantia nigra and by the presence of intracytoplasmic inclusions called Lewy bodies. ${ }^{43,44}$

Mitochondrial dysfunction plays an essential role in PD pathogenesis, both in sporadic forms and in those of hereditary origin, and can be caused by bioenergetic defects, mitochondrial DNA mutations, nuclear DNA mutations linked to mitochondrial genes, changes in the fusion or fission processes and mitochondrial traffic alterations. One of the proposed reasons for the vulnerability of substantia nigra dopaminergic neurons is that they basally contain lower numbers of mitochondria in comparison with other midbrain neurons. ${ }^{45}$ Mitochondrial dynamics alterations have been reported to increase ROS production and decrease mitochondrial membrane potential, which 
could potentiate the accumulation of dysfunctional mitochondria. However, excessive mitochondrial fragmentation has also been associated with sporadic PD pathology.

In a study that compared samples of patients with idiopathic PD and animals exposed to paraquat (an herbicide used as a model of the disease), an increase in Drp1 expression and a reduction in Mfn1/Mfn2 expression in the substantia nigra were observed, accompanied by an increase in mitochondrial fragmentation and dysfunction. By overexpressing Mfn2, mitochondrial fragmentation was eliminated and neurons were protected against paraquat-induced dysfunction. Paraquat-induced oxidative damage and dopaminergic neurons selective loss in the substantia nigra and in axonal terminals in the striatum were also observed to decrease in transgenic mice that overexpressed hMfn2. ${ }^{46}$

Alterations in the OPA1 structure have been shown to be related to the mitochondrial fragmentation observed in sporadic PD cellular models, which is associated with an increase in Drp1 expression. To demonstrate the relevance of these alterations, OPA1 was overexpressed, and it inhibited Drp1 expression. In this study, OPA1 overexpression did not modify mitochondrial fragmentation, and neither did it decrease ROS levels, but an increase was found in mitochondrial elongation and interconnectivity, which maintained the membrane potential. ${ }^{47}$

On the other hand, the brains of patients with PD have diminished mitochondrial respiratory capacity and biogenesis, which might induce $\beta$-amyloid plaqueand neurofibrillary tangle formation. In addition, the ROS generated by dysfunctional mitochondrial networks can activate the signaling pathways that induce cell death, whereas aggregate proteins contribute to neuroinflammation. ${ }^{48}$

Mutations in the Parkin protein have been associated with early-onset autosomal recessive PD. In studies with C57BL adult mice, Parkin decrease in the middle ventral region of the brain induced age-dependent dopaminergic neuron loss. This process is subject to the accumulation of the Parkin substrate called PARIS, also known as zinc finger protein 746 (ZNF746), and to PGC-1 $\alpha$ transcriptional repression. In Parkin-knockout mice, mitochondria size and number was decreased in the ventral midbrain of the C57BL adult mouse, which was prevented through PARIS silencing. On the other hand, PARIS overexpression in the ventral midbrain of mice decreased PGC-1 $\alpha$ activation and the number of mitochondria. These same results were demonstrated in the SH-SY5Y cell line. Together, these data suggest that Parkin loss affects mitochondrial biogenesis, which decreases the respiratory function due to a lower number of mitochondria and decreased cell death. ${ }^{49}$

Mitochondrial and lysosomal function are known to decrease with age, ${ }^{50.51}$ which agrees with the fact that age is the most important risk factor for the development of PD. Mitophagy and lysosomal activity deterioration have been associated with the $\alpha$-synuclein increased expression observed in PD. ${ }^{51,52}$ The NRF2 and TFEB transcription factors that play a role in mitochondrial and lysosomal biogenesis, respectively, can regulate p62/SQSMT1, a protein that marks damaged mitochondria for their degradation by mitophagy. Cells with higher levels of the TFEB protein have been reported to have higher PGC-1 $\alpha$ mRNA expression levels. Therefore, the strategies to increase TFEB have been proposed to be able to increase the number of mitochondria and prevent mitochondrial dysfunction in PD..$^{53}$

\section{Huntington's disease}

Huntington's disease (HD) is a pathology that affects some people in the middle of their life. It is genetic in nature, with autosomal dominant inheritance. $\mathrm{HD}$ is characterized by motor dysfunction, involuntary movements, dystonia, cognitive impairment and emotional disorders..$^{54,55}$ This disease has a low prevalence, since it affects between 10 and 13 people per 100,000 population..$^{56}$ The main areas of the brain that are affected include the caudate and putamen of the striatum, the cerebral cortex, the hippocampus, the hypothalamus and subthalamus. ${ }^{54,57}$ Neuronal decrease in these regions of the brain has been observed in up to $80 \%$ of patients with severe HD..$^{58}$

$\mathrm{HD}$ is caused by repeated expansion of unstable CAG trinucleotides in exon 1 of the Huntingtin gene $(\mathrm{Htt})$ that encodes a mutant form of the huntingtin protein $(\mathrm{Htt})$. The presence of more than $40 \mathrm{CAG}$ repetitions is recognized as the mutant huntingtin ( $\mathrm{mHtt}$ ), which causes the disease. The onset of HD generally occurs in the median age, followed by 15 to 20 years of disease progression. ${ }^{59}$

Different lines of research support the hypothesis that alteration of mitochondrial and energetic functions is important in the HD pathophysiology. ${ }^{60-62} \mathrm{Sim}$ ilar to other NDs, mitochondrial fission was shown to be increased in samples of patients with different degrees of HD, since Drp1 levels were increased and those of Mfn1 were decreased. A decrease in PGC-1 $\alpha$ and TFAM expression has also been reported. ${ }^{62}$ 
Shirendeb et al. confirmed these results: Fis1 and Drp1 were found to be increased, while Opa1, Mfn1, Mfn2 and Tom40 were decreased in patients with grade III and IV HD in comparison with patients without the disease.$^{63}$ In primary cultures of BACHD mice neurons expressing $\mathrm{mHtt}$, as well as in samples of patients with HD, the increase in Drp1-dependent mitochondrial fragmentation was found to be due to its interaction with $\mathrm{mHtt}$. An increase in Drp1 and Fis1 levels of expression was also observed, as well as a Mfn1/Mfn2 decrease. ${ }^{63}$

Alterations in mitochondrial dynamics, such as imbalance between fission and fusion can be offset to a certain extent by an increase in mitochondrial biogenesis. However, $\mathrm{mHtt}$ has been reported to decrease mitochondrial function by inhibiting PGC-10 expression. ${ }^{64-66} \mathrm{mHtt}$ represses PGC-1 $\alpha$ gene transcription by binding to the promoter and interfering with the CREB/TAF4-dependent transcriptional pathway, which critically participates in the expression of PGC-10-regulated genes. PGC- $1 \alpha$ overexpression has been found to partially reverse $\mathrm{mHtt}$ toxic effects in primary striatal neuron cultures, and PGC- $1 \alpha$ lentivirus-mediated administration into the striatum was shown ro provide neuroprotection in HD transgenic mice. ${ }^{64}$

On the other hand, the removal of defective mitochondria has been suggested to be compromised in $H D$. The elimination of defective mitochondria depends on the mitophageal activity that involves the PINK1/Parkin pathway. ${ }^{67-69}$ Recently, failures in the mitophagy process were reported in flies that expressed $\mathrm{mHtt}$, such as the Htt-ex1p-Q93 neuronal models, as well as in the HdhQ111 striatal cell line, which are associated with decreased ubiquitinated proteins in mitochondria that are to be degraded in autophagosomes. ${ }^{70}$ The same study showed that PINK1 overexpression worked as neuroprotector in flies and restored mitophagy in HdhQ111 cells. Wild-type Htt has been proposed to play a physiological role in mitophagy, possibly due to its interaction with autophagy receptors. Specifically, Htt carboxyl terminal was reported to interact with p62 to facilitate the recognition of proteins that have to be ubiquitinated, which is relevant to mitophagy, since Htt-deficient cells have lower mitophagy levels. ${ }^{71}$ Mitochondrial transport along the microtubules also decreases in primary cultures of $\mathrm{mHtt}$-expressing cortical neurons, ${ }^{72.73}$ since the $\mathrm{mHtt}$ aggregates can block the transport to axons, sequester motor proteins and inhibit the formation of mitochondrial traffic complexes. ${ }^{73.74}$

\section{Conclusion}

Reports on the relationship of mitochondrial dynamics with NDs propose a new field of research for the development of alternative treatments that reduce symptoms and deterioration. The works herein described show interesting results, since overexpression of proteins associated with mitochondrial dynamics, in particular of fusion proteins, is encouraging, since improvements in energy supply and mitochondrial transport, as well as in fragmentation decrease, have been observed. However, the challenge for future research will lie in the development of mechanisms that prevent interactions of proteins that are aberrantly expressed in NDs with proteins that participate in mitochondrial dynamics and reduce mitochondrial damage, in order to provide patients with a better quality of life.

\section{Acknowledgments}

This work was supported by Conacyt funding CB2015-01-255591 granted to Norma Edith López-Diazguerrero ${ }^{1}$ and FON.INST/298/2016 received by Mina Königsberg, as well as by Conacyt's Red Temática de Investigación en Salud y Desarrollo Social.

\section{References}

1. Grimm A, Eckert A. Brain aging and neurodegeneration: from a mitochondrial point of view. J Neurochem. 2017;143:418-431.

2. Shah SZA, Zhao D, Hussain T, Yang L. Role of the AMPK pathway in promoting autophagic flux via modulating mitochondrial dynamics in neurodegenerative diseases: insight into prion diseases. Ageing Res Rev. 2017;40:51-63.

3. Chen Y, Zhang H, Zhou HJ, Ji W, Min W. Mitochondrial redox signaling and tumor progression. Cancers (Basel). 2016;8:40.

4. Frohman MA. Role of mitochondrial lipids in guiding fission and fusion. J Mol Med (Berl). 2015;93:263-269.

5. Gao J, Wang L, Liu J, Xie F, Su B, Wang X. Abnormalities of mitochondrial dynamics in neurodegenerative diseases. Antioxidants (Basel). 2017;6:25.

6. Golpich M, Amini E, Mohamed Z, Azman Ali R, Mohamed Ibrahim N, Ahmadiani A. Mitochondrial dysfunction and biogenesis in neurodegenerative diseases: pathogenesis and treatment. CNS Neurosci Ther. 2017;23:5-22.

7. Labbé K, Murley A, Nunnari J. Determinants and functions of mitochondrial behavior. Annu Rev Cell Dev Biol. 2014;30:357-391.

8. Wai T, Langer T. Mitochondrial dynamics and metabolic regulation. Trends Endocrinol Metab. 2016;27:105-117.

9. Youle RJ, van Der-Bliek AM. Mitochondrial fission, fusion, and stress. Science. 2012;337:1062-1065.

10. Lackner LL, Nunnari JM. The molecular mechanism and cellular functions of mitochondrial division. Biochim Biophys Acta. 2009;1792:1138-1144.

11. Jahn R, Scheller RH. SNAREs engines for membrane fusion. Nat Rev Mol Cell Biol. 2006;7:631-643.

12. Meeusen SL, Nunnari J. How mitochondria fuse. Curr Opin Cell Biol. 2005; 17:389-394.

13. Hoppins S, Nunnari J. The molecular mechanism of mitochondrial fusion. Biochim Biophys Acta. 2009;1793:20-26.

14. Pernas L, Scorrano L. Mito-morphosis: mitochondrial fusion, fission, and cristae remodeling as key mediators of cellular function. Annu Rev Physiol. 2016:78:505-531.

15. López-Lluch G. Mitochondrial activity and dynamics changes regarding metabolism in ageing and obesity. Mech Ageing Dev. 2017;162:108-121.

16. Horbay R, Bilyy R. Mitochondrial dynamics during cell cycling. Apoptosis. 2016;21:1327-1335.

17. Chan DC. Mitochondria: dynamic organelles in disease, aging, and development. Cell. 2006;125:1241-1252. 
18. Campello S, Scorrano L. Mitochondrial shape changes: Orchestrating cell pathophysiology. EMBO Rep. 2010;11:678-684.

19. Friedman JR, Nunnari J. Mitochondrial form and function. Nature. 2014;16;505:335-343

20. Cho DH, Nakamura T, Lipton SA. Mitochondrial dynamics in cell death and neurodegeneration. Cell Mol Life Sci. 2010;67:3435-3447.

21. Hoppins S. The regulation of mitochondrial dynamics. Curr Opin Cell Biol. 2014:29:46-52.

22. Ventura-Clapier R, Garnier A, Veksler V. Transcriptional control of mitochondrial biogenesis: the central role of PGC-1alpha. Cardiovasc Res. 2008;79:208-217.

23. Scarpulla RC. Nucleus-encoded regulators of mitochondrial function: integration of respiratory chain expression, nutrient sensing and metabolic stress. Biochim Biophy Acta. 2012;1819:1088-1097.

24. Kang Y, Fielden LF, Stojanovski D. Mitochondrial protein transport in health and disease. Semin Cell Dev Biol. 2018:76:142-153.

25. Austin S, St Pierre J. PGC1 $\alpha$ and mitochondrial metabolism: emerging concepts and relevance in ageing and neurodegenerative disorders. J Cell Sci. 2012:125:4963-4971.

26. Scarpulla RC. Metabolic control of mitochondrial biogenesis through the PGC1 family regulatory network. Biochim Biophys Acta. 2011;1813:1269-1278.

27. Campello S, Strappazzon F, Cecconi F. Mitochondrial dismissal in mammals, from protein degradation to mitophagy. Biochim Biophys Acta. 2014; $1837: 451-460$

28. Wen-Xing Ding, Xiao-Ming Y. Mitophagy: mechanisms, pathophysiological roles, and analysis. Biol Chem. 2012;393:547-564.

29. Dolman NJ, Chambers KM, Mandavilli B, Batchelor RH, Janes MS. Tools and techniques to measure mitophagy using fluorescence microscopy. Autophagy. 2013;9:1653-1662.

30. Hamacher-Brady A, Brady NR. Mitophagy programs: mechanisms and physiological implications of mitochondrial targeting by autophagy. Cell Mol Life Sci. 2016;73:775-795.

31. Correia SC, Perry G, Moreira PI. Mitochondrial traffic jams in Alzheimer's disease: pinpointing the roadblocks. Biochim Biophys Acta. 2016; 1862:1909-1917.

32. Swomley AM, Förster S, Keeney JT, Triplett J, Zhang Z, Sultana R, et al. Abeta, oxidative stress in Alzheimer disease: evidence based on proteomics studies. Biochim. Biophys Acta. 2014;1842:1248-1257.

33. Smith MA. Alzheimer disease. Int Rev Neurobiol. 1998;42:1-54

34. Wang X, Su B, Lee HG, Li X, Perry G, Smith MA, et al. Impaired balance of mitochondrial fission and fusion in Alzheimer's disease. J Neurosci. 2009;29:9090-9103.

35. Manczak M, Reddy PH. Abnormal interaction between the mitochondria fission protein Drp1 and hyperphosphorylated tau in Alzheimer's disease neurons: implications for mitochondrial dysfunction and neuronal damage. Hum Mol Genet. 2012;21:2538-2547.

36. Baek SH, Park SJ, Jeong JI, Kim SH, Han J, Kyung JW, et al. Inhibition of Drp1 ameliorates synaptic depression, $A \beta$ deposition, and cognitive impairment in an Alzheimer's disease model. J Neurosci. 2017; 37:5099-5110.

37. Kandimalla R, Manczak M, Yin X, Wang R, Reddy PH. Hippocampa phosphorylated tau induced cognitive decline, dendritic spine loss and mitochondrial abnormalities in a mouse model of Alzheimer's disease. Hum Mol Genet. 2018;27:30-40

38. Sheng B, Wang X, Su B, Lee HG, Casadesus G, Perry G, et al. Impaired mitochondrial biogenesis contributes to mitochondrial dysfunction in Alzheimer's disease. J Neurochem. J Neurochem. 2012:120:419-429.

39. Hu Y, Li XC, Wang ZH, Luo Y, Zhang X, Liu XP, et al. Tau accumulation impairs mitophagy via increasing mitochondrial membrane potential and reducing mitochondrial Parkin. Oncotarget. 2016;7:17356-17368.

40. Wang L, Guo L, Lu L, Sun H, Shao M, Beck SJ, et al. Synaptosoma mitochondrial dysfunction in 5xFAD mouse model of Alzheimer's disease. PLoS One. 2016;11:e0150441.

41. Yan MH, Wang X, Zhu X. Mitochondrial defects and oxidative stress in Alzheimer disease and Parkinson disease. Free Radic Biol Med. 2013;62:90-101.

42. Sepúlveda-Falla D, Barrera-Ocampo A, Hagel C, Korwitz A Vinueza-Veloz MF, Zhou K, et al. Familial Alzheimer's disease-associated presenilin-1 alters cerebellar activity and calcium homeostasis. J Clin Invest. 2014;124:1552-1567.

43. Alexander GE. Biology of Parkinson's disease: pathogenesis and pathophysiology of a multisystem neurodegenerative disorder. Dialogues Clin Neurosci. 2004;6:259-280.

44. Dauer W, Przedborski S. Parkinson's disease: mechanisms and models. Neuron. 2003:39:889-909.

45. Liang CL, Wang TT, Luby-Phelps K, German DC. Mitochondria mass is low in mouse substantianigra dopamine neurons: implications for Parkinson's disease. Exp Neurol. 2007;203:370-380.

46. Zhao F, Wang W, Wang C, Siedlak SL, Fujioka H, Tang B, et al. Mfn2 protects dopaminergic neurons exposed to paraquat both in vitro and in vivo: implications for idiopathic Parkinson's disease. Biochim Biophys Acta Mol Basis Dis. 2017:1863:1359-1370.
47. Santos D, Esteves AR, Silva DF, Januário C, Cardoso SM. The impact of mitochondrial fusion and fission modulation in sporadic Parkinson's disease. Mol Neurobiol. 2015;52:573-586.

48. Onyango IG, Khan SM, Bennett JP. Mitochondria in the pathophysiology of Alzheimer's and Parkinson's diseases. Front Biosci (Landmark Ed). 2017;22:854-872.

49. Stevens DA, Lee Y, Kang HC, Lee BD, Lee YI, Bower A, et al. Parkin oss leads to PARIS-dependent declines in mitochondrial mass and respiration. Proc Natl Acad Sci U S A. 2015;112:11696-11701.

50. Terman A, Kurz T, Navratil M, Arriaga EA, Brunk UT. Mitochondrial turnover and aging of long-lived postmitotic cells: the mitochondrial-lysosomal axis theory of aging. Antioxid Redox Signal. 2010;12:503-535.

51. Nixon RA. The role of autophagy in neurodegenerative disease. Nat Med. 2013;19:983-997.

52. Schapira AH, Gegg M. Mitochondrial contribution to Parkinson's disease pathogenesis. Parkinsons Dis. 2011;2011:159160.

53. Ivankovic D, Chau KY, Schapira AH, Gegg ME. Mitochondrial and biogenesis are activated following PINK1/parkin-mediated mitophagy. Jeurochem. 2016;136:388-402.

54. Colpo GD, Stimming EF, Rocha NP, Teixeira AL. Promises and pitfalls of immune-based strategies for Huntington's disease. Neural Regen Res. 2017;12:1422-1425.

55. Montoya A, Price BH, Menear M, Lepage M. Brain imaging and cognitive dysfunctions in Huntington's disease. J Psychiatry Neurosci. 2006; 31:21-29.

56. McColgan P, Tabrizi SJ. Huntington's disease: a clinical review. Eur J Neurol. 2018;25:24-34

57. Waldvogel HJ, Kim EH, Thu DC, Tippett LJ, Faull RL. New perspectives on the neuropathology in huntington's disease in the human brain and its relation to symptom variation. J Huntingtons Dis. 2012;1:143-153.

58. Vonsattel JP, Myers RH, Stevens TJ, Ferrante RJ, Bird ED, Richardson EP. Neuropathological classification of Huntington's disease. J Neuropathol Exp Neurol. 1985;44:559-577.

59. Langbehn DR, Hayden MR, Paulsen JS, PREDICT-HD Investigators of the Huntington Study Group. CAG-repeat length and the age of onset in Huntington disease (HD): a review and validation study of statistical approaches. Am J Med Genet B Neuropsychiatr Genet. 2010;153:397-408.

60. Browne SE, Beal MF. The energetics of Huntington's disease. Neurochem Res. 2004:29:531-546.

61. Beal MF. Mitochondria take center stage in aging and neurodegeneration. Ann Neurol. 2005;58:495-505

62. Kim J, Moody JP, Edgerly CK, Bordiuk OL, Cormier K, Smith K, et al. Mitochondrial loss, dysfunction and altered dynamics in Huntington's disease. Hum Mol Genet. 2010;19:3919-3935.

63. Shirendeb UP, Calkins MJ, Manczak M, Anekonda V, DufourB, McBride JL, et al. Mutant huntingtin's interaction with mitochondrial protein Drp1 impairs mitochondrial biogenesis and causes defective axonal transport and synaptic degeneration in Huntington's disease. Hum Mol Genet. 201;21:406-420.

64. Cui L, Jeong H, Borovecki F, Parkhurst CN, Tanese N, Krainc D. Transcriptional repression of PGC-1alpha by mutant hunting in leads to mitochondrial dysfunction and neurodegeneration. Cell. 2006; 127:59-69.

65. Weydt P, Pineda VV, Torrence AE, Libby RT, Satterfield TF, Lazarowski ER, et al. Thermoregulatory and metabolic defects in Huntington's disease transgenic mice implicate PGC-1alpha in Huntington's disease neurodegeneration. Cell Metab. 2006;4:349-362.

66. Chaturvedi RK, Calingasan NY, Yang L, Hennessey T, Johri A, Beal MF. Impairment of PGC-1alpha expression, neuropathology and hepatic steatosis in a transgenic mouse model of Huntington's disease following chronic energy deprivation. Hum Mol Genet. 2010;19:3190-3205.

67. Pickrell AM, Youle RJ. The roles of PINK1, Parkin, and mitochondrial fidelity in Parkinson's disease. Neuron. 2015:85:257-273.

68. Ashrafi G, Schwarz TL. PINK1- and PARK2-mediated local mitophagy in distal neuronal axons. Autophagy. 2015;11:187-189.

69. Wang X, Winter D, Ashrafi G, Schlehe J, Wong YL, Selkoe D, et al. PINK1 and Parkin target Miro for phosphorylation and degradation to arrest mitochondrial motility. Cell. 2011;147:893-906.

70. Khalil B, El-Fissi N, Aouane A, Cabirol-Pol MJ, Rival T, Liévens JC. PINK1-induced mitophagy promotes neuroprotection in Huntington's disease. Cell Death Dis. 2015;22;6:e1617

71. Rui YN, Xu Z, Patel B, Chen Z, Chen D, Tito A, et al. Huntingtin functions as a scaffold for selective macroautophagy. Nat Cell Biol. 2015;17:262-275.

72. Chang DT, Rintoul GL, Pandipati S, Reynolds IJ. Mutant huntingtin aggregates impair mitochondrial movement and trafficking in cortical neurons. Neurobiol Dis. 2006;22:388-400.

73. Orr AL, Li S, Wang CE, Li H, Wang J, Rong J, et al. N-terminal mutant huntingtin associates with mitochondria and impairs mitochondrial trafficking. J Neurosci. 2008;28:2783-2792.

74. Oliveira JM. Nature and cause of mitochondrial dysfunction in Huntington's disease: focusing on huntingtin and the striatum. J Neurochem. 2010;114:1-12. 